\title{
A new coagulation defect associated with a case of melanomatosis
}

\author{
J. D. PHILLIPS AND M. J. O'SHEA \\ From the Department of Pathology, Mytton Oak Road, Shrewsbury SY3 $8 X H$
}

SUMMARY A marked abnormality of the intrinsic coagulation system was observed in a patient with melanomatosis and frank melanuria. With successful treatment of the tumour there was a parallel improvement in the clotting abnormality. This defect was shown to be a deficiency of factor XI together with a previously unrecognised factor. This is distinct from either Fletcher or Fitzgerald factors and appears to act between factors XI and X in the coagulation sequence.

Liver disease is well known to be associated with coagulation abnormalities, particularly of the 'prothrombin complex' and fibrinogen. Coagulation tests before liver biopsy often show abnormalities of factors II, VII, IX, and X which may revert to normal after vitamin $K_{1}$ therapy. Screening tests of the intrinsic and extrinsic systems usually reflect these abnormalities to a similar degree in each test. Subnormal levels of factor XI are also associated with liver pathology (Nossel, 1972). A case is reported with a mild prolongation of the prothrombin time yet a marked abnormality in the activated partial thromboplastin time, not fully explained by a deficiency of either factors IX or XI.

\section{Clinical history}

A 31-year-old woman presented with massive hepatomegaly, generalised pigmentation and melanuria. Two years previously a malignant melanoma had been excised from the left leg. There was no history of excessive bleeding during surgery or childbirth, nor of any more recent haemorrhagic manifestations. There was no bleeding tendency in her immediate family; both parents, an only brother, and two sons were all alive and well. Routine coagulation screening tests were carried out and showed a normal blood count and platelet numbers but a markedly prolonged activated partial thromboplastin time (APTT) with a mild prolongation of the prothrombin time. Further coagulation investigations were carried out following this initial observation to elucidate the nature of the defect. Treatment for the melanoma with four 10-day courses of dacarbazine has resulted in good clinical

Received for publication 15 November 1976 improvement with reduction in liver size, loss of melanuria, and a return to normal of the coagulation changes.

\section{Coagulation studies}

Standard techniques were used for the routine investigations. British Comparative Thromboplastin with the fibrometer system (Baltimore Biological Laboratory, Maryland, USA) was used for the prothrombin time estimation. APTT were performed manually using Dade activated cephaloplastin which contains ellagic acid and employs a 2 minute incubation. The effect of a longer incubation period of 8 minutes was also measured. Factors VIII and IX were determined in one-stage assays using known deficient human plasma (Dade, American Hospital Supply (UK) Ltd, Didcot, Oxfordshire). Factor V was assayed in a one-stage prothrombin time using normal oxalated plasma depleted in factor V. Factor I was assayed using Dade Data-Fi reagents and the fibrometer system. The whole blood clotting time was performed using the method of Lee and White, and the bleeding time using Ivy's method. Adsorbed plasma was prepared by incubating 9 volumes of normal plasma with 1 volume of BDH moist alumina gel $(25 \mathrm{~g} / \mathrm{dl})$ at $37^{\circ} \mathrm{C}$ for 3 minutes, followed by centrifugation to deposit the aluminium hydroxide, and removal of the supernatant. The depletion of factors II, VII, IX, and X was confirmed by checking the prothrombin time of the adsorbed plasma. Normal aged serum was prepared by allowing normal blood to clot in a glass tube at $37^{\circ} \mathrm{C}$ for one hour, followed by centrifugation and removal of the serum and further incubation of the serum for 6 hours. The product was stored at $-20^{\circ} \mathrm{C}$ until used. An initial 
factor XI assay was performed using an artificially prepared substrate plasma, as described by Blewitt (1975), and later assays on stored plasmas using Dade bovine congenital factor XI deficient plasma.

Contact product was prepared as follows: $2 \mathrm{ml}$ of platelet poor plasma was transferred to a plastic tube and $1 \mathrm{ml}$ of Koch-Light celite 512 suspension (100 $\mathrm{g} / 1$ in $0.85 \mathrm{~g} / \mathrm{dl} \mathrm{NaCl}$ ) was added. The tube was transferred to a $37^{\circ} \mathrm{C}$ waterbath and incubated for 10 minutes with constant agitation, followed by centrifugation to deposit the celite. The supernatant was removed and replaced with the original plasma volume of $0.85 \mathrm{~g} / \mathrm{dl} \mathrm{NaCl}$. The celite suspension was mixed, centrifuged, and washed five times in $0.85 \mathrm{~g} / \mathrm{dl}$ $\mathrm{NaCl}$. Then $2 \mathrm{ml}$ of $10 \mathrm{~g} / \mathrm{dl} \mathrm{NaCl}$ were added to the celite, and the mixture was transferred to the $37^{\circ} \mathrm{C}$ bath and agitated for 10 minutes. The celite was removed by centrifugation and the eluate dialysed for 12 hours against 10 times its own volume of distilled water. Finally, aliquots of the eluate were stored at $-20^{\circ} \mathrm{C}$ until required. The partial thromboplastin time with contact product (PTT/CP) was performed by incubating $0.2 \mathrm{ml}$ plasma and $0.2 \mathrm{ml}$ cephalin (from KCCT method) at $37^{\circ} \mathrm{C}$ for 55 seconds, then adding $0.2 \mathrm{ml} \mathrm{M} / 40 \mathrm{CaCl}_{2}$ followed by $0.1 \mathrm{ml}$ contact product at 1 minute. Clot formation was timed from the addition of contact product. Kaolin cephalin clotting times (KCCT) were performed by incubating $0.2 \mathrm{ml}$ diluting fluid, $0.2 \mathrm{ml}$ plasma, $0.2 \mathrm{ml}$ cephalin, and $0.1 \mathrm{ml}$ prewarmed kaolin $(0.5 \mathrm{~g} / \mathrm{dl})$ at $37^{\circ} \mathrm{C}$, tilting the tube each minute to mix. After 10 minutes' incubation $0.2 \mathrm{ml}$ of $0.025 \mathrm{M} \mathrm{CaCl}_{2}$ was added and clot formation was timed. The cephalin was supplied by the National Reference Laboratory for Anticoagulant Control Reagents and after reconstitution was diluted 1:50 in Owren's buffer for use. The diluting fluid was prepared by adding $200 \mathrm{ml}$ Owren's buffer to $200 \mathrm{ml} 0.025 \mathrm{M}$ sodium citrate and making up to 1 litre with $0.85 \mathrm{~g} / \mathrm{dl} \mathrm{NaCl}$.

\section{Results}

The results of the initial coagulation studies and the changes subsequent to treatment of the melanomatosis are shown in Table 1 . The major abnormality is seen in the APTT. This abnormality was totally corrected in mixture experiments with normal plasma or aged normal serum, and partially corrected by adsorbed normal plasma. An incubated screening test excluded the presence of an inhibitor.

Since the prolongation of the prothrombin time was very mild (thought to be secondary to liver disease), the major coagulation defect reflected in the APTT was assumed to act at a level above factor $X$ in the sequence. Assays of factors VIII and IX were normal and therefore a contact factor deficiency was suspected. The Lee and White whole blood clotting time $\left(8 \frac{1}{2} \mathrm{~min}\right)$ was normal and this excluded factor XII deficiency, therefore factor XI deficiency was suspected. The Ivy bleeding time was 3 minutes; this is variable but usually normal in contact factor deficiencies. The marked abnormality shown in the APTT was present to a lesser degree in the KCCT. This was found to be independent of the differing incubation times of the two tests.

STUDIES USING CONCENTRATE FROM URINE This patient was shown to be excreting large amounts of melanin in the urine using the sodium nitroprusside and ferric chloride tests. The larger molecules, including melanin, present in the urine were concentrated using Visking tubing, and the contents of the tubing were centrifuged to concentrate the melanin. The supernatant was removed leaving a brown precipitate. On the addition of glyoxalin buffer $(\mathrm{pH}$ 7.3) a colour change from brown to black occurred and the precipitate went into solution. One volume of this solution was mixed with 9 volumes of normal plasma and incubated for 3 minutes at $37^{\circ} \mathrm{C}$. This preparation had an APTT of 140 seconds and factor XI assay of $27 \%$. This compared with an artificially prepared substrate plasma (Blewitt, 1975) with an APTT of 168 seconds, factor XI $1.6 \%$. The plasma was further incubated with urine concentrate for 1 hour at $37^{\circ} \mathrm{C}$, and mixtures with normal plasma and patients' plasma were tested. The results are shown in Table 2.

Concentrates of normal urine tested in the same manner failed significantly to prolong the APTT of normal plasma.

EXPERIMENTS WITH CONTACT PRODUCT Contact product was prepared from normal plasma. This product, tested against known factor deficient plasmas, detected defects acting later than factor XI but was insensitive to factor XI deficiency. Plasma from the patient (day 5 and day 30) was also used to prepare contact product. The contact product from these three sources and plasmas from the same three sources were tested in a partial thromboplastin time, using contact product as activator (PTT/CP), with the object of investigating the stage in the coagulation mechanism at which the defect in this patient acts. The results are recorded in Table 3.

Any possible additive effect of the low factor XI reflected in the two components of the reaction ('bridge effect') when using weak contact product was excluded by comparing the effect of a range of dilutions of normal contact product on normal plasma, and on plasma containing $10 \%$ of factor XI. The various contact product dilutions gave similar results with each of the two plasmas. 


\section{Discussion}

Routine screening tests before liver biopsy revealed a minimal prolongation of the prothrombin time and a major abnormality in the intrinsic coagulation mechanism revealed by the activated partial thromboplastin time. It was concluded that the abnormality was acting in the clotting sequence at a stage before the activation of factor $\mathrm{X}$, and the presence of an inhibitor as a possible cause of the prolongation of the APTT was excluded. A normal whole blood clotting time excluded a significantly low level of factor XII. Factor VIII and IX levels were normal, and assays of factor XI during the period of observation varied from $21 \%$ to $75 \%$ while over the same period the APTT varied from 266 to 37 seconds. The impression that such levels of factor XI as $21 \%$ and $46 \%$ would not lead to prolongation of the APTT to 266 and 280 seconds was confirmed by the observation that artificially prepared factor XI deficient plasma with a level of only $1.6 \%$ was prolonged to a lesser degree in the APTT (168 seconds) than the patient's plasma. It therefore seemed that this patient had a dual deficiency involving factor XI and another 'factor' outside the accepted sequence of reactions in the coagulation mechanism.

It was shown that treating normal plasma with a concentrate from the patient's urine appeared to induce a defect similar to that of the patient, since the patient's plasma failed to correct the defect in the altered plasma. Normal plasma partly corrected this defect. It is postulated that the melanin concentrated from the patient's urine, or another substance with a molecular weight of greater than approximately 10000 retained in the visking tubing, may have had some adsorptive properties in respect of factors which contribute to clotting in normal plasma.

Prolonging the contact phase incubation period in the APTT test did not appear significantly to correct the defect. This appears to exclude a deficiency of Fletcher factor which is said to be associated with correction of the APTT on prolonged incubation (Hathaway and Alsever, 1970; Hattersley and Hayse, 1976).

Furthermore, reagents incorporating ellagic acid as an activator of factor XII may be relatively insensitive to the defect in Fletcher factor deficiency (Abildgaard and Harrison, 1974). One feature was the shorter KCCT obtained using this patient's plasma in comparison with the APTT results using Dade cephaloplastin. This was initially thought to be due to the longer incubation period $(10 \mathrm{~min})$ of the KCCT, but prolonging the incubation period of the APTT has excluded this. Thus, it would seem that kaolin as an activator of the contact factors has a differing effect from ellagic acid in this defect. The
Table 1 Initial coagulation studies (day 1-2) and after cytotoxic therapy (day 5-53)

\begin{tabular}{|c|c|c|c|c|c|c|c|}
\hline \multirow[t]{2}{*}{ Investigation } & \multicolumn{7}{|l|}{ Day } \\
\hline & 1 & 2 & 5 & 8 & 13 & 30 & 53 \\
\hline PT (normal $13 \mathrm{~s}$ ) & 16 & 17 & & 15 & & & 15 \\
\hline $\begin{array}{l}\text { APTT (normal } 37 \mathrm{~s} \text { ) } \\
\text { KCCT (normal } 45 \mathrm{~s} \text { ) }\end{array}$ & 320 & 266 & $\begin{array}{r}310 \\
95\end{array}$ & 280 & $\begin{array}{r}110 \\
62\end{array}$ & 35 & $\begin{array}{l}37 \\
46\end{array}$ \\
\hline Factor I (mg/dl) & & 450 & 445 & & 340 & 280 & 215 \\
\hline Factor V \% & & & & 75 & & & \\
\hline Factor VIII \% & & 100 & & & & & \\
\hline Factor IX \% & & 100 & & & & & \\
\hline Factor XI \% & & 21 & 33 & 46 & 40 & 64 & 75 \\
\hline
\end{tabular}

Table 2 Experiments using concentrate from urine excreted by the patient

$\operatorname{APTT}(s)$

\begin{tabular}{lr}
\hline Altered plasma (3 min incubation of plasma and & \\
urine extract) & 140 \\
Altered plasma (60 min incubation) & 130 \\
Normal untreated plasma & 32 \\
$50: 50$ altered plasma + normal plasma & 75 \\
1: 9 altered plasma + normal plasma & 36 \\
$50: 50$ altered plasma + patient's plasma & 140 \\
1: 9 altered plasma + patient's plasma & 140 \\
$50: 50$ buffer + patient's plasma & 240 \\
$50: 50$ normal plasma + patient's plasma & 40 \\
\hline
\end{tabular}

Table 3 Experiments using contact product as activator in the cephalin clotting time

\begin{tabular}{llll}
\hline \begin{tabular}{l} 
Source of $\begin{array}{l}\text { contact product } \\
\end{array}$ \\
\cline { 2 - 4 }
\end{tabular} & \multicolumn{3}{l}{ Cephalin clotting time $(s)$ with contact product } \\
\cline { 2 - 4 } & Source of plasma \\
\cline { 2 - 4 } & Normal & Test day 30 & Test day 5 \\
\hline Normal & 46 & 57 & 65 \\
Test day 30 & 48 & 58 & 77 \\
Test day 5 & 55 & 66 & 105 \\
\hline
\end{tabular}

absence of any major prolongation of the Lee and White whole blood clotting time appears to exclude deficiency of Fitzgerald (Flaujeac) factor, although features such as the results obtained in the KCCT and APTT, and mixture experiments, are similar to those obtained in the original patient said to be Flaujeac factor deficient (Lacombe et al., 1975; Waldmann and Abraham, 1974).

Both the postulated unrecognised factors known as Fletcher and Fitzgerald factors appear to act before factor XI. Partial thromboplastin times with contact product were performed to establish whether the described defect affects the APTT before or after the action of factor XI. Contact product is a material with an activity which corresponds to the activation of contact clotting factors XI and XII and thus converts factor IX to its activated product directly. Controls confirmed that the contact product 
prepared from normal plasma was sensitive to deficiency of factor IX but insensitive to factor XI deficiency. Contact product was prepared from two samples of the patient's plasma and would be expected to show some inefficiency in that prepared from the plasma of day 5 since the factor XI was rather low at that time. However, the primary defect presented in the patient's plasma appears to act later than factor XI because the times are more prolonged using the patient's plasma as a substrate, than when normal plasma is used as a substrate, for any given source of contact product.

Thus, it is suggested that this patient had a transient deficiency of both factor XI and a previously unrecognised factor which interacts with factor XI in the activation of factor IX, or acts at a later stage before the activation of factor $\mathrm{X}$ by factor VIII. This observation was associated with gross hepatomegaly associated with melanomatosis, and one possible cause is that interaction had occurred between the melanin pigments and the deficient coagulation factors.

\section{References}

Abildgaard, C. F. and Harrison, J. (1974). Fletcher factor deficiency: family study and detection. Blood, 43, 641644.

Blewitt, R. E. (1975). A new method for the assay of factor $\mathrm{XI}$ in plasma and the preparation and use of a new artificial substrate. Journal of Clinical Pathology, 28, 332336.

Hathaway, W. E. and Alsever, J. (1970). The relation of "Fletcher factor" to factors XI and XII. British Journal of Haematology, 18, 161-169.

Hattersley, P. G. and Hayse, D. (1976). The effect of in- $\overrightarrow{\vec{C}}$ creased contact activation time on the activated partial ${ }^{\circ}$ thromboplastin time. American Journal of Clinical $\overrightarrow{0}$ Pathology, 66, 479-482.

Lacombe, M. J., Varet, B., and Levy, J. P. (1975). A hitherto undescribed plasma factor acting at the con- 9 tact phase of blood coagulation (Flaujeac factor): case of report and coagulation studies. Blood, 46, 761-768.

Nossel, H. L. (1972). The contact system. In Human Blood 윽 Coagulation Haemostasis and Thrombosis, edited by R. Biggs, pp. 79-132. Blackwell, Oxford.

Waldmann, R. and Abraham, J. P. (1974). Fitzgerald factor: A heretofore unrecognised coagulation factor (Abstract). Blood, 44, 934. 